ARTICLE

\title{
Nanoscale imaging of bacterial infections by sphingolipid expansion microscopy
}

\author{
Ralph Götz ${ }^{1,4}$, Tobias C. Kunz ${ }^{2,4}$, Julian Fink ${ }^{3}$, Franziska Solger ${ }^{2}$, Jan Schlegel @ ${ }^{1}$, Jürgen Seibel (10 ${ }^{3}$,
} Vera Kozjak-Pavlovic (iD ${ }^{2}$, Thomas Rudel (i) ${ }^{2 \otimes} \&$ Markus Sauer (i) ${ }^{1 \times}$

Expansion microscopy (ExM) enables super-resolution imaging of proteins and nucleic acids on conventional microscopes. However, imaging of details of the organization of lipid bilayers by light microscopy remains challenging. We introduce an unnatural short-chain azide- and amino-modified sphingolipid ceramide, which upon incorporation into membranes can be labeled by click chemistry and linked into hydrogels, followed by $4 \times$ to $10 \times$ expansion. Confocal and structured illumination microscopy (SIM) enable imaging of sphingolipids and their interactions with proteins in the plasma membrane and membrane of intracellular organelles with a spatial resolution of $10-20 \mathrm{~nm}$. As our functionalized sphingolipids accumulate efficiently in pathogens, we use sphingolipid ExM to investigate bacterial infections of human HeLa229 cells by Neisseria gonorrhoeae, Chlamydia trachomatis and Simkania negevensis with a resolution so far only provided by electron microscopy. In particular, sphingolipid ExM allows us to visualize the inner and outer membrane of intracellular bacteria and determine their distance to $27.6 \pm 7.7 \mathrm{~nm}$.

\footnotetext{
${ }^{1}$ Department of Biotechnology and Biophysics, Biocenter, Julius-Maximilians-Universität Würzburg, Am Hubland, 97074 Würzburg, Germany. ${ }^{2}$ Department of Microbiology, Biocenter, Julius-Maximilians-Universität Würzburg, Am Hubland, 97074 Würzburg, Germany. ${ }^{3}$ Institute for Organic Chemistry, JuliusMaximilians-Universität Würzburg, Am Hubland, 97074 Würzburg, Germany. ${ }^{4}$ These authors contributed equally: Ralph Götz, Tobias C. Kunz.

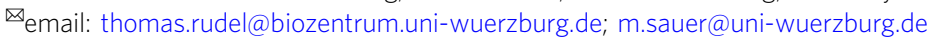


$\mathrm{n}$ the last decade, super-resolution microscopy has evolved as a very powerful method for subdiffraction-resolution fluorescence imaging of cells and structural investigations of cellular organelles ${ }^{1,2}$. Super-resolution microscopy methods can now provide a spatial resolution that is well below the diffraction limit of light microscopy, enabling invaluable insights into the spatial organization of proteins in biological samples. However, in particular three-dimensional and multicolor super-resolution microscopy methods require elaborate equipment and experience and are therefore mostly restricted to specialized laboratories.

Expansion microscopy (ExM) provides an alternative approach to bypass the diffraction limit and enable super-resolution imaging on standard fluorescence microscopes. By linking a protein of interest into a dense, cross-linked network of a swellable polyelectrolyte hydrogel, biological specimens can be physically expanded allowing $\sim 70 \mathrm{~nm}$ lateral resolution by confocal laser scanning microscopy. Since its introduction by Boyden and coworkers in $2015^{3}$, expansion microscopy (ExM) has shown impressive results including the magnified visualization of pre- or post-expansion labeled proteins and RNAs with fluorescent proteins, antibodies, and oligonucleotides, respectively, in cells, tissues, and human clinical specimen ${ }^{4}$. ExM has been developing at a high speed with various protocols providing expansion factors from $4 x^{3}$ to $10 x^{5,6}$ and even $20 x$ by iterative expansion ${ }^{7}$. In addition, various protocols have been introduced enabling subdiffraction-resolution imaging of proteins, RNA, and bacteria in cultured cells, neurons, and tissues by confocal fluorescence microscopy and in combination with super-resolution microscopy $^{5-14}$.

In order to be usable for ExM, the molecule of interest has to exhibit amino groups that can react with glutaraldehyde $(\mathrm{GA})^{9}$, MA-NHS ${ }^{9}$, AcX ${ }^{10}$, or Label- $\mathrm{X}^{11}$ and be linked into the polyelectrolyte hydrogel. The plasma membrane of cells is mainly composed of glycerophospholipids, sphingolipids, and cholesterol. Due to the lack of primary amino groups, these lipids neither can be fixed by formaldehyde, glutaraldehyde and other chemical fixatives nor expanded using available ExM protocols. To this end, we sought to functionalize a lipid that is compatible with ExM. So far, sphingolipids have only been functionalized as azides to enable fluorescence labeling by click chemistry after incorporation into cellular membranes ${ }^{15-18}$. Therefore, we set out to introduce an azide and primary amino group into sphingolipids to enable fluorescence labeling and chemical fixation as well as linking of the lipid into a swellable hydrogel. Our results demonstrate that the designed unnatural bifunctional sphingolipid is efficiently incorporated into membranes of cells and accumulates in bacterial membranes, which allowed us to investigate the distribution of lipids and interactions with proteins in cellular and bacterial membranes with high spatial resolution.

\section{Results}

Sphingolipid ExM of cellular membranes. Sphingolipids are natural lipids comprised of the sphingoid base backbone sphingosine, which when $\mathrm{N}$-acylated with fatty acids forms ceramide, a central molecule in sphingolipid biology. Sphingolipid ceramides regulate cellular processes such as differentiation, proliferation, growth arrest and apoptosis. Ceramide-rich membrane areas promote structural changes within the plasma membrane, which segregate membrane receptors and affect the membrane curvature and vesicle formation, fusion and trafficking ${ }^{19,20}$.

In a previous study, we showed that functionalized long-chain $\omega-\mathrm{N}_{3}-\mathrm{C}_{16}$-ceramide is incorporated into cellular membranes but cannot be efficiently click-labeled with DBCO-functionalized dyes because of the hindered accessibility of the $\omega$ - $\mathrm{N}_{3}$-group after membrane incorporation ${ }^{21}$. In contrast, unnatural short-chain $\omega$ $\mathrm{N}_{3}-\mathrm{C}_{6}$-ceramide is efficiently incorporated into cellular membranes and can be click-labeled with DBCO-functionalized dyes for fluorescence imaging ${ }^{21-23}$. Therefore, we selected the unnatural $\omega-\mathrm{N}_{3}-\mathrm{C}_{6}$-ceramide for further functionalization with a primary amino group (Supplementary Figs. 1-13) and synthesized $\alpha-\mathrm{NH}_{2}-\omega-\mathrm{N}_{3}-\mathrm{C}_{6}$-ceramide from (tert-butoxycarbonyl)-L-lysine (Fig. 1a). We first assessed if the synthesized $a-\mathrm{NH}_{2}-\omega-\mathrm{N}_{3}-\mathrm{C}_{6}$-ceramide (Fig. 1a) is incorporated into cellular membranes similar to the control ceramide without amino modification and can be labeled by click chemistry with DBCOdyes. For this, cells were fed for $1 \mathrm{~h}$ with the two ceramides, fixed with glutaraldehyde and click-labeled with DBCO-Alexa Fluor 488. Confocal fluorescence images showed that both analogs $\omega$ $\mathrm{N}_{3}-\mathrm{C}_{6}$-ceramide and $\alpha-\mathrm{NH}_{2}-\omega-\mathrm{N}_{3}-\mathrm{C}_{6}$-ceramide are incorporated into the plasma membrane and membranes of intracellular organelles of HeLa229 cells (Fig. 1). Fluorescence recovery after photobleaching (FRAP) experiments with both ceramides indicated that $\omega-\mathrm{N}_{3}-\mathrm{C}_{6}$-ceramide shows a higher mobility in the plasma membrane after fixation than $\alpha-\mathrm{NH}_{2}-\omega-\mathrm{N}_{3}-\mathrm{C}_{6}$-ceramide (Fig. 1b). This finding was corroborated by the treatment of labeled cells with detergents, which wash out unfixed lipids. Upon addition of Triton X-100 or saponin $\omega-\mathrm{N}_{3}-\mathrm{C}_{6}$-ceramide was efficiently washed out whereas the fluorescence signal of the amino-modified analog $\alpha-\mathrm{NH}_{2}-\omega-\mathrm{N}_{3}-\mathrm{C}_{6}$-ceramide decreased only slightly and was preserved for weeks (Fig. 1c and Supplementary Fig. 14). These results demonstrate that the crosslinker glutaraldehyde can fix amino-modified ceramides incorporated into cellular membranes.

Since glutaraldehyde (GA) can link proteins into hydrogels ${ }^{9}$ we reasoned that $\alpha-\mathrm{NH}_{2}-\omega-\mathrm{N}_{3}-\mathrm{C}_{6}$-ceramides might be as well suited for membrane expansion. To demonstrate its usefulness for ExM we treated HeLa229 with $\mathrm{NH}_{2}-\omega-\mathrm{N}_{3}-\mathrm{C}_{6}$-ceramide followed by glutaraldehyde fixation, permeabilization, fluorescence labeling with DBCO-Alexa Fluor 488, and gelation. For direct comparison we tested the membrane-binding fluorophore-cysteine-lysinepalmitoyl group (mCling), which labels the plasma membrane and is taken up during endocytosis ${ }^{24}$. Since it carries a primary amine as well, it also remains attached to membranes after fixation and permeabilization and can therefore potentially be used for ExM. In fact, both amino-functionalized membrane probes can be expanded using the GA ExM protocol ${ }^{9} .4 \times$ and $10 \times$ expanded confocal fluorescence images of ceramide stained cells showed staining of the plasma membrane as well as of membranes of intracellular organelles such as mitochondria, whereas mCling is efficiently incorporated mainly into the cell's plasma membrane (Fig. 2a). To verify the expansion factor and investigate if sphingolipid ExM distorts membranes we imaged the same cell before and after $4 \times$ and $10 \times$ expansion and determined effective expansion factors of $4.1 \times$ and $9.8 \times$, respectively (Supplementary Fig. 15). The confocal fluorescence images of $4 \times$ and $10 \times$ expanded cellular membranes demonstrate that sphingolipid ExM labeling is dense enough to support nanoscale resolution imaging of continuous membrane structures and even thin membrane protrusions (Fig. 2 and Supplementary Fig. 15).

Imaging of expanded lipids and proteins. Furthermore, we tested if the sphingolipid ExM protocol enables imaging of lipids and proteins in the same sample. We therefore immunolabeled the mitochondrial matrix protein Peroxiredoxin 3 (Prx3) after permeabilization and click labeling of the unnatural short-chain bifunctional ceramide. Peroxiredoxins are antioxidant enzymes that also control cytokine-induced peroxide levels and mediate signal transduction. Prx3 is exclusively located in the 
a

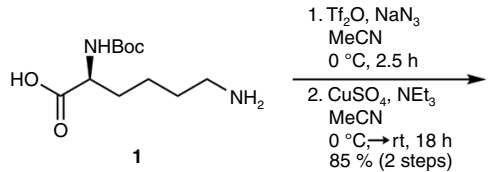

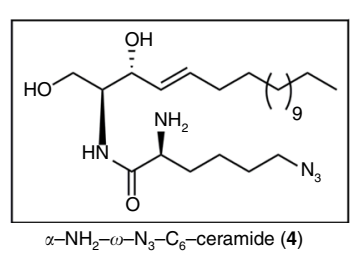
TFA
$\mathrm{CH}_{2} \mathrm{Cl}_{2}$
$0^{\circ} \mathrm{C}, 2 \mathrm{~h}$
$39 \%$<smiles>CCCCCC(N)C(=O)O</smiles>
Sphingosine HATU, $\mathrm{NEt}_{3}$ DMF $0^{\circ} \mathrm{C}, \rightarrow \mathrm{rt}, 4 \mathrm{~h}$
$48 \%$

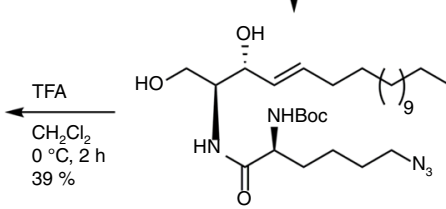

3

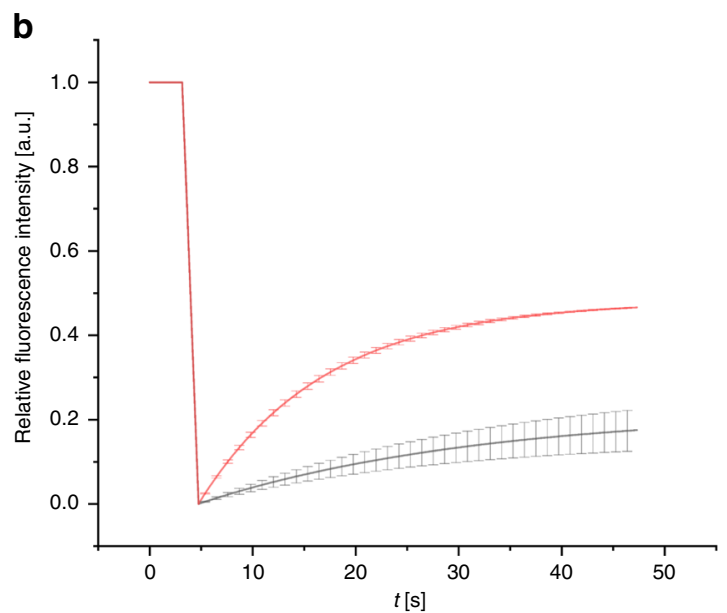

$[\mathrm{s}]$
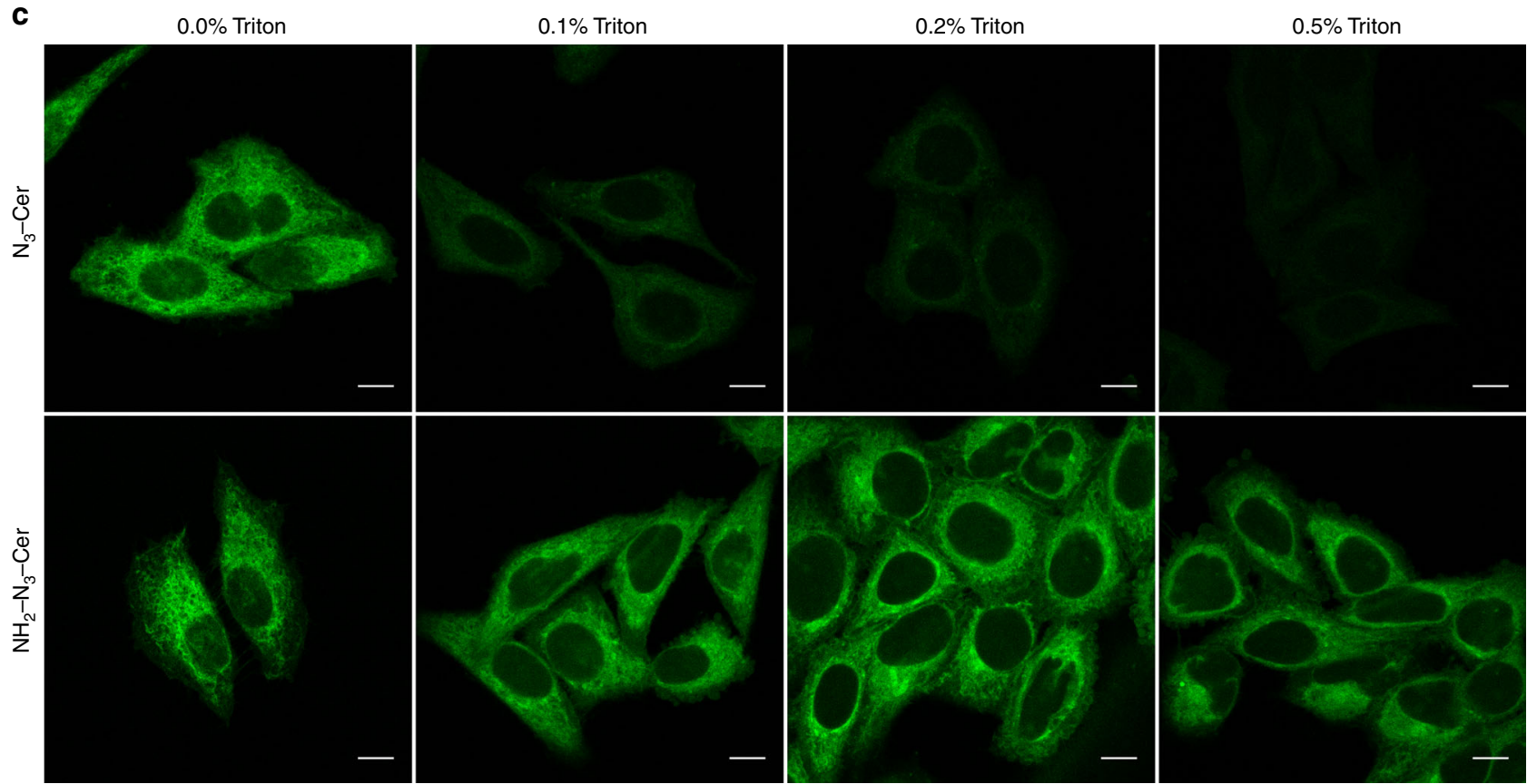

Fig. 1 Amino- and azido-functionalized sphingolipids enable fixation and fluorescence labeling of lipids. a Schematic overview of the synthesis of $\alpha-\mathrm{NH}_{2}-\omega-\mathrm{N}_{3}-\mathrm{C}_{6}$-ceramide (for synthesis details see Material and Methods and Supporting Information). To investigate the mobility of membraneincorporated functional sphingolipids HeLa229 cells were fed with $10 \mu \mathrm{M} \alpha-\mathrm{NH}_{2}-\omega-\mathrm{N}_{3}-\mathrm{C}_{6}$-ceramide or $\omega$ - $\mathrm{N}_{3}$ - $\mathrm{C}_{6}$-Ceramide, fixed, permeabilized and stained with DBCO-Alexa Fluor 488. b FRAP experiments with the two incorporated ceramide analogs. After three confocal fluorescence imaging frames, a circular region of interest with a diameter $1.8 \mu \mathrm{m}$ was bleached and fluorescence recovery followed over time. The $\alpha-\mathrm{NH}_{2}-\omega-\mathrm{N}_{3}-\mathrm{C}_{6}-\mathrm{ceramide}(\mathrm{black})$ shows a lower mobility (mean mobile fraction of $22.2 \%$ ) than the $\omega-\mathrm{N}_{3}-\mathrm{C}_{6}$-ceramide (red) lacking the primary amino group (mobile fraction of $48.1 \%$ ). Source data are provided as Source Data file. c Confocal fluorescence images of fixed and labeled cells in the presence of increasing concentrations of the detergent TritonX100. With increasing Triton-X100 concentration $\omega-\mathrm{N}_{3}-\mathrm{C}_{6}$-ceramide $\left(\mathrm{N}_{3}-\mathrm{Cer}\right)$ is efficiently washed out while the $\alpha-\mathrm{NH}_{2}-\omega-\mathrm{N}_{3}-\mathrm{C}_{6}-\mathrm{ceramide}\left(\mathrm{NH} \mathrm{H}_{2}-\mathrm{N}_{3}-\mathrm{Cer}\right)$ signal remains preserved. The data were obtained from $n=2$ independent experiments. Scale bars, $10 \mu \mathrm{m}$.

mitochondrial membrane. Specific immunostaining of Prx3 is not compromised by the incorporation of the amino-functionalized sphingolipid $\mathrm{NH}_{2}-\omega-\mathrm{N}_{3}-\mathrm{C}_{6}$-ceramide into the mitochondrial membrane (Fig. 2b). These results show that the aminofunctionalized sphingolipid $\mathrm{NH}_{2}-\omega-\mathrm{N}_{3}-\mathrm{C}_{6}$-ceramide can be used for super-resolution imaging of cellular membranes and in combination with immunostaining for visualization of interactions between proteins and ceramides in $4 \mathrm{x}$ and 10x expanded samples. Very recently, Boyden and coworkers introduced an alternative membrane ExM method (mExM) based on a membrane intercalating probe, which enables imaging of $4.5 \mathrm{x}$ expanded cellular membranes ${ }^{25}$. The membrane probe contains a chain of lysines for binding to a polymer anchorable handle and a lipid tail on the amine terminus of the lysine chain, with a glycine in between to provide mechanical flexibility. Furthermore, a biotin residue is attached to enable fluorescence staining of the probe with labeled streptavidin. Alternatively, a trifunctional linker strategy termed TRITON has been introduced for ExM that enables simultaneous targeting, labeling, and grafting of biomolecules using a monomer unit (acryloyl) into the hydrogel $^{26}$. Using 1,2-distearoyl-sn-glycero-3-phosphoethanolamine (DSPE) as small-molecule targeting staining phospholipid bilayers, cellular membranes can be expanded and imaged. All these alternative methods can be used as well for joint imaging of proteins and lipid membrane structures at nanoscale resolution. 


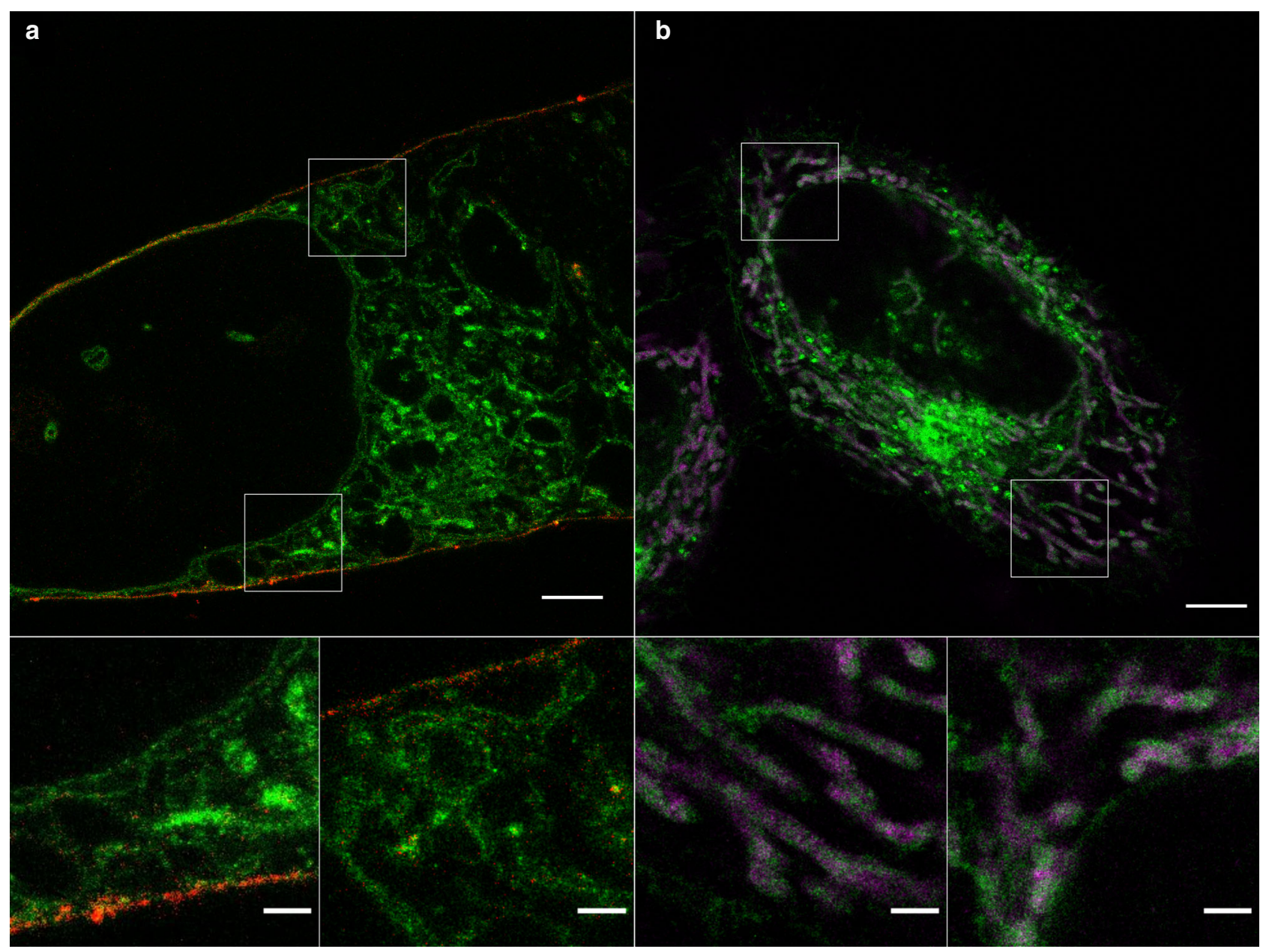

Fig. 2 Sphingolipid ExM enables super-resolution imaging of cellular membranes and protein interactions. a Confocal fluorescence image of a 10x expanded HeLa229 cell fed with ATTO643-mCling (red) and $\alpha-\mathrm{NH}_{2}-\omega-\mathrm{N}_{3}-\mathrm{C}_{6}$-ceramide clicked with DBCO-Alexa Fluor 488 (green). Scale bars, $20 \mu$ m. The images at the bottom show magnified views of the regions outlined by the white boxes in the main images. $\mathbf{b}$ Confocal fluorescence image of $4 \mathrm{x}$ expanded HeLa229 cells. Cells were fed with $\alpha-\mathrm{NH}_{2}-\omega-\mathrm{N}_{3}-\mathrm{C}_{6}$-ceramide, fixed, permeabilized, and labeled with DBCO-Alexa Fluor 488 (green). In addition, Prx3 (magenta), which is located in the mitochondrial matrix was stained by immunolabeling using ATTO $647 \mathrm{~N}$ labeled secondary antibodies. The data were obtained from $n=3$ independent experiments. Scale bars, $5 \mu \mathrm{m}$.

Sphingolipid ExM of bacterial infections. In addition to the regulation of cellular processes, ceramides play an essential role in infections with pathogenic bacteria ${ }^{27,28}$. These include Neisseria gonorrhoeae ${ }^{29}$, Simkania negevensis ${ }^{30}$ and Chlamydia trachomatis ${ }^{31,32}$. C. trachomatis is by far the best investigated example for an interaction of pathogenic bacterium and host sphingolipid metabolism. This obligate intracellular Gramnegative bacterium is the most frequent cause of bacterial sexually transmitted diseases ${ }^{33}$. It resides in a membrane-bound vacuole (the inclusion) inside their host cells and undergoes a complex developmental cycle between infectious non-replicating elementary bodies (EB) and non-infectious replicating reticulate bodies (RB). During infection, Chlamydia manipulate a plethora of cellular processes, among them the sphingolipid metabolism $^{15,16,34}$. The ceramide transporter CERT seems to play a key role in ceramide uptake as it strongly localizes in infected cells at the inclusion membrane recruited by the bacterial inclusion protein IncD instead of mediating golgi-ER-trafficking ${ }^{35}$.

To investigate the uptake of short-chain ceramides by pathogens during infection we first fed cells with $\mathrm{NH}_{2}-\omega-\mathrm{N}_{3}$ $\mathrm{C}_{6}$-ceramide for 5 to $60 \mathrm{~min} 24 \mathrm{~h}$ post infection with $C$. trachomatis. The cells were then GA fixed and click-labeled with DBCO-Alexa Fluor 488 for fluorescence imaging. Confocal fluorescence images demonstrated rapid integration of the unnatural ceramide into the membrane of C. trachomatis already after $5 \mathrm{~min}$ and further increasing for longer incubation times (Supplementary Fig. 16). This indicates effective and fast ceramide uptake by C. trachomatis. Additionally, we applied the specific CERT inhibitor HPA-12 to impede ceramide integration into the bacterial membrane. Fluorescence images recorded after application of HPA-12 showed that HPA-12 inhibits ceramide uptake by $C$. trachomatis at higher concentrations for short incubation times of 5 and 15 min (Supplementary Fig. 16). For longer incubation times the influence of HPA-12 treatment on ceramide uptake by bacteria was negligible, suggesting the involvement of different lipid uptake pathways such as vesicle trafficking from the Golgi apparatus ${ }^{36}$.

Since the loss of lipopolysaccharide (LPS) has dramatic effects on the viability of many Gram-negative bacteria and was shown to inhibit the development of chlamydial infectious elementary bodies $^{37}$, we tested if treatment with unnatural $\alpha-\mathrm{NH}_{2}-\omega-\mathrm{N}_{3}-\mathrm{C}_{6}-$ ceramide results in the replacement of chlamydial LPS in the outer bacterial membrane. Upon incorporation of $\alpha-\mathrm{NH}_{2}-\omega-\mathrm{N}_{3}$ $\mathrm{C}_{6}$-ceramide, we could not detect strong differences in the amount of LPS compared to untreated samples (Supplementary Fig. 17). Moreover, sphingolipids are known to exert toxic effects 
on bacteria in vitro ${ }^{18,38}$ and in vivo ${ }^{39}$. We therefore investigated, if exposure of Chlamydia to $\alpha-\mathrm{NH}_{2}-\omega-\mathrm{N}_{3}-\mathrm{C}_{6}$-ceramide affects their capacity to form inclusions or infectious progeny reminiscent of an intact developmental cycle. Both, formation of inclusions and infectious progeny was unaffected in $\alpha-\mathrm{NH}_{2-} \omega$ $\mathrm{N}_{3}-\mathrm{C}_{6}$-ceramide treated cells (Supplementary Fig. 18), demonstrating that the incorporation of short-chain unnatural ceramides does not have a major impact on chlamydial viability. Chlamydia trachomatis incorporated $\alpha-\mathrm{NH}_{2}-\omega-\mathrm{N}_{3}-\mathrm{C}_{6}$-ceramide even when the cells were fed before infection, indicating the direct uptake of short-chain ceramides from the host (Supplementary Fig. 18a). The addition of $a-\mathrm{NH}_{2}-\omega-\mathrm{N}_{3}-\mathrm{C}_{6}$-ceramide before infection, continuously during infection or before fixation neither influenced chlamydial development nor the infectivity of chlamydial progeny (Supplementary Figs. 18b, c). Feeding $\alpha-$ $\mathrm{NH}_{2}-\omega-\mathrm{N}_{3}-\mathrm{C}_{6}$-ceramides directly before fixation resulted in the highest incorporation efficiency (Supplementary Fig. 18a). Cytotoxicity assays with $\alpha-\mathrm{NH}_{2}-\omega-\mathrm{N}_{3}-\mathrm{C}_{6}$-ceramide showed that $1 \mathrm{~h}$ of treatment does not induce cytotoxic effects in HeLa229 cells (Supplementary Fig. 19).

Next, we investigated if the uptake of short-chain unnatural ceramides by intracellular pathogens enables ExM of infected cells. Therefore, we fed $\mathrm{NH}_{2}-\omega-\mathrm{N}_{3}-\mathrm{C}_{6}$-ceramide to HeLa229 cells post-infection with $C$. trachomatis and $S$. negevensis, another member of the order Chlamydiales (Fig. 3). Cells were then fixed with GA, permeabilized, click-labeled with DBCO-Alexa Fluor 488 and expanded using two different ExM protocols. Confocal fluorescence images of the same cells recorded before and after $10 \mathrm{x}$ expansion revealed a good quality agreement of bacterial membrane shapes and numbers of bacteria (Supplementary Fig. 20). In addition, the post-expansion images showed that the ceramides accumulate strongly in bacterial membranes after infection (Supplementary Fig. 20).

Cells infected with a high number of $S$. negevensis required $10 \mathrm{x}$ expansion to distinguish individual bacteria (Fig. 3a-c). On the other hand, already $4 \mathrm{x}$ expansion was sufficient to distinguish between the two forms of $C$. trachomatis, RBs and EBs as has already been shown previously by ExM (Fig. 3d, e) ${ }^{8}$. Higher expansion (10x ExM) demonstrated that the ceramide signal accumulates in the membranes of the two pathogens $C$. trachomatis and $S$. negevensis (Fig. 3c, f). The fluorescence signals of host cell membranes appeared comparably dim under identical experimental settings (compare Fig. 2 and Fig. 3) indicating efficient uptake of short-chain functionalized ceramides by bacteria. Corresponding control experiments with $\omega-\mathrm{N}_{3}-\mathrm{C}_{6}-$ ceramide and DBCO-Alexa Fluor 488 alone showed only very weak background staining (Supplementary Fig. 21). These results show that sphingolipid ExM enables continuous membrane staining of intracellular bacteria and thus imaging of bacterial infections by Neisseria gonorrhoeae, Chlamydia trachomatis and Simkania negevensis.

Furthermore, we tested if the ceramide underlying structure sphingosine can be used successfully for ExM. The sphingoid base backbone sphingosine carries a natural amino group and plays a central role in infections with $N$. gonorrhoeae among other bacterial pathogens ${ }^{36}$. Addition of $\omega-\mathrm{N}_{3}$-sphingosine to infected Chang cells followed by GA fixation, permeabilization, click labeling with DIBO-Alexa Fluor 488 and gelation demonstrated the general applicability of the method. Details of intracellular $N$. gonorrhoeae can be clearly visualized by sphingolipid ExM (Supplementary Fig. 22).

Imaging interactions of bacteria and intracellular proteins. To demonstrate the compatibility of sphingolipid ExM for investigations of pathogen interactions with intracellular proteins, we investigated chlamydial interactions with mitochondria. It is known that $C$. trachomatis reorganizes the host organelles. However, so far all investigations have been performed by confocal fluorescence imaging or electron microscopy ${ }^{40}$. Hence, we immunolabeled the mitochondrial matrix protein Prx3 and incorporated ceramides in C. trachomatis infected cells before gelation. The corresponding confocal fluorescence images of $10 \mathrm{x}$ expanded samples showed the mitochondrial rearrangement after infection with C. trachomatis as mitochondria localized around the inclusion (Fig. 3g). To highlight details of this interaction by a higher spatial resolution we used structured illumination microscopy (SIM) ${ }^{41}$, which allowed us to uncover direct interactions between mitochondria and C. trachomatis (Fig. 3h). In some cases, Prx 3 signals appeared to be located in bacteria indicating unspecific protein uptake. Similar experiments performed in the absence of primary antibodies demonstrated that the signals detected in bacteria are not caused by nonspecific binding of the used secondary antibody (Supplementary Fig. 23). Albeit shortchain ceramides accumulate in bacterial membranes the labeling density of intracellular membranes is still high enough to enable nanoscale imaging of protein-pathogen interactions in infected cells.

Interestingly, we could often detect individual Chlamydia within close proximity to the inclusion membrane after feeding with $\mathrm{NH}_{2}-\omega-\mathrm{N}_{3}-\mathrm{C}_{6}$-ceramides, possibly indicating an active docking to the inclusion membrane and an absorption of nutrition by $C$. trachomatis (Supplementary Fig. 24 and Supplementary Movie 1) as has been hypothesized earlier ${ }^{42}$ and reported in electron microscopy studies ${ }^{40,43}$. This behavior has previously been proposed as a mechanism by which RBs acquire nutrients including host lipids ${ }^{44}$ and as an essential step in chlamydial development ${ }^{42}$. However, previous attempts to localize chlamydial particles in the inclusion required highly laborious techniques such as Serial block-face scanning electron microscopy ${ }^{40}$. Using sphingolipid ExM with clickable probes, the three-dimensional structure of lipid interfaces can be imaged at a lateral resolution of $\sim 20 \mathrm{~nm}$ by confocal fluorescence microscopy.

10x Sphingolipid ExM-SIM resolves the double membrane of intracellular bacteria. Whereas transport of ceramide to the Chlamydia inclusion has been reported earlier ${ }^{31}$, one of the unanswered questions is whether ceramides form parts of the bacterial outer (OM) or inner membrane (IM) or of both these membranes. Indeed, SIM images of $10 \mathrm{x}$ expanded Chlamydia demonstrated that unnatural $\mathrm{NH}_{2}-\omega-\mathrm{N}_{3}-\mathrm{C}_{6}$-ceramides are efficiently incorporated into the IM and OM of intracellular Chlamydia (Fig. 4a, b). With a doubling of the spatial resolution provided by SIM and the high labeling density of ceramides, $10 \mathrm{x}$ expanded samples can be imaged with an estimated spatial resolution of $10-20 \mathrm{~nm}$ enabling us to resolve the IM and OM of gram-negative bacteria. We investigated three different infected cells and selected those bacteria whose orientation allowed us to visualize spatially separated OM and IM (i.e., frontal views of bacteria) and determined the distance between the two membranes to $27.6 \pm 7.7 \mathrm{~nm}$ (s.d.) from 23 cross sectional intensity profiles (Supplementary Figs. 25 and 26). This value is typical for the separation of OM and IM of gram-negative bacteria and in agreement with electron microscopy data ${ }^{45}$. Since the mechanism of bacterial membrane biogenesis from host-derived lipids is currently unknown, our finding of unnatural ceramide incorporation in both bacterial membranes suggests an active process rather than only the fusion of lipid vesicles with the surface and exclusive integration into the outer membrane of Chlamydia.

The high spatial resolution provided by sphingolipid ExM may also be used to study mechanisms of antibiotic resistance. 


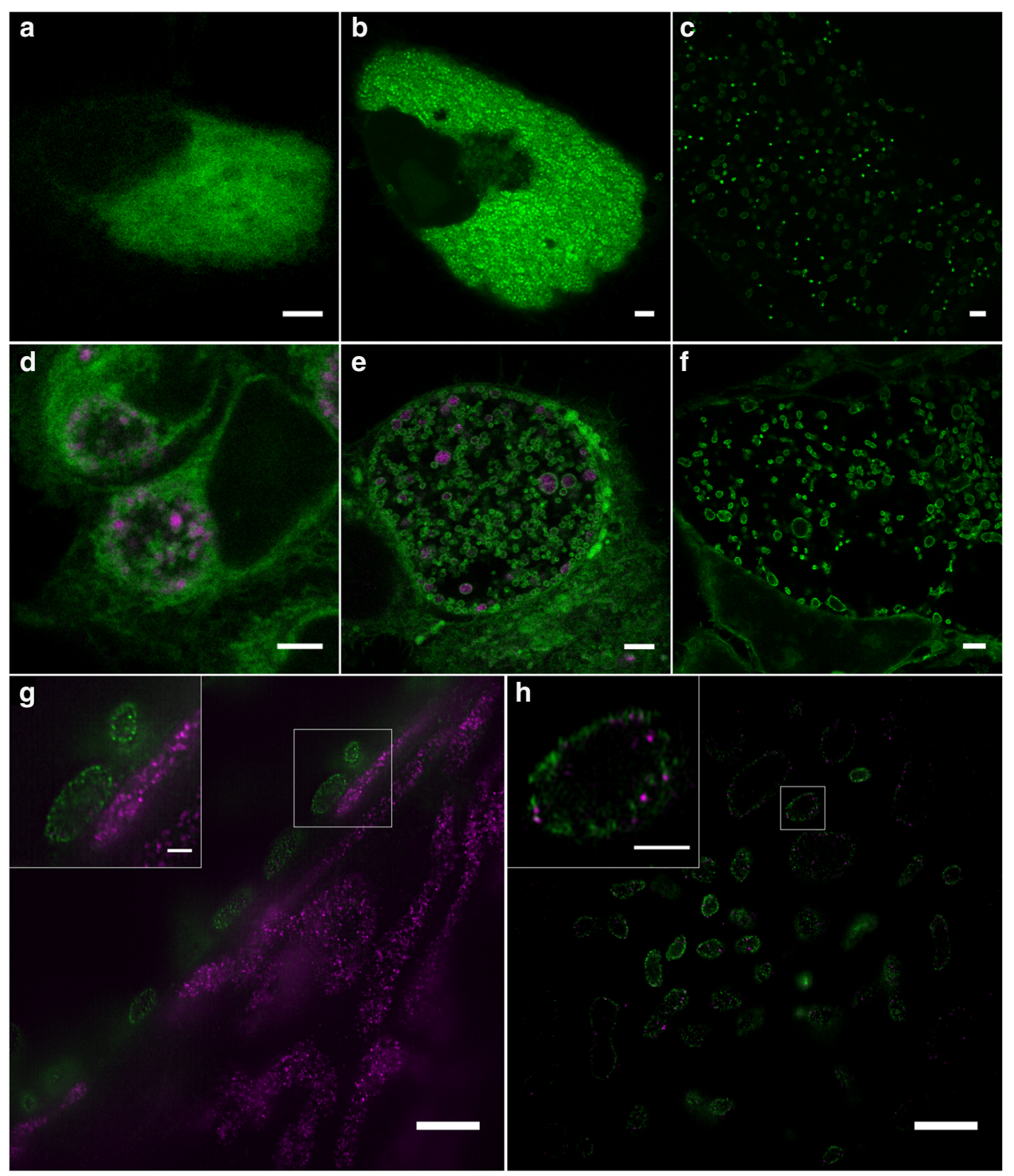

Fig. 3 Sphingolipid ExM visualizes intracellular pathogens and their interactions with mitochondrial proteins. a-c Cells were infected with Simkania negevensis for $96 \mathrm{~h}$, fed with $\alpha-\mathrm{NH}_{2}-\omega-\mathrm{N}_{3}-\mathrm{C}_{6}$-ceramide, fixed, permeabilized and stained with DBCO-Alexa Fluor 488 (green), and then imaged. The images show different cells before expansion (a), after $4 x$ expansion (b), and 10x expansion (c) recorded by confocal microscopy. $\mathbf{d}$-f Cells were infected with Chlamydia trachomatis for $24 \mathrm{~h}$, fed with $\alpha-\mathrm{NH}_{2}-\omega-\mathrm{N}_{3}-\mathrm{C}_{6}$-ceramide, fixed, permeabilized and stained with DBCO-Alexa Fluor 488 (green). Different cells were imaged before expansion (d), after $4 x$ expansion (e), and 10x expansion (f) by confocal microscopy. In the unexpanded (d) and $4 x$ expanded image (e) chlamydial HSP60 was immunolabeled with ATTO647N secondary antibody (magenta). $\mathbf{g}$ The mitochondrial marker protein Prx3 was stained by immunolabeling with an ATTO 647N secondary antibody (magenta). The confocal fluorescence image of 10x expanded samples revealed a close contact between Chlamydia and mitochondria at the inclusion membrane. $\mathbf{h}$ SIM images of 10x expanded samples uncover that some Prx3 molecule are inserted into the bacterial membrane. The data were obtained from $n=3$ independent experiments. Scale bars, $5 \mu \mathrm{m}$ (unexpanded images $\mathbf{a}$, $\mathbf{d}$ ), $10 \mu \mathrm{m}$ ( $4 \mathrm{x}$ and $10 \mathrm{x}$ expanded images $\mathbf{b}, \mathbf{c}, \mathbf{e}, \mathbf{f}, \mathbf{g}, \mathbf{h}$ ), and $2 \mu \mathrm{m}$ (magnified views in images $\mathbf{g}, \mathbf{h}$ ).

Infections with multidrug-resistant gram-negative bacteria are difficult to treat because of the double membrane that is impermeable for most antibiotics ${ }^{46}$. Hence, being able to visualize the double membrane might promote the development of antibiotics with improved membrane permeability. Furthermore, sphingolipid ExM might also be useful for the investigation of ceramide pathways related to apoptosis, proliferation, cancer, inflammation, and neurodegeneration ${ }^{37,47}$.

\section{Discussion}

ExM has facilitated super-resolution imaging of cells and tissues with standard fluorescence microscopes available in most research facilities, yet it has been limited to the expansion of proteins and nucleic acids due to the lack of primary amino groups in lipids. We have developed the double-functionalized unnatural sphingolipid $\mathrm{NH}_{2}-\omega-\mathrm{N}_{3}-\mathrm{C}_{6}$-ceramide that incorporates efficiently into cellular and bacterial membranes and can be fixed, fluorescently labeled by click chemistry, and linked into polyelectrolyte hydrogels by GA treatment. Our study included bacterial infections of human HeLa229 cells by Neisseria gonorrhoeae, Chlamydia trachomatis and Simkania negevensis. The applicability of the method to other bacterial strains has to be tested in further experiments. The mechanism by which GA fixes and crosslinks amino-modified ceramides into hydrogels is less obvious but most probably associated with the existence of multimeric forms of GA containing aldehyde and alkene groups, which both can potentially be covalently linked to the acrylamide 


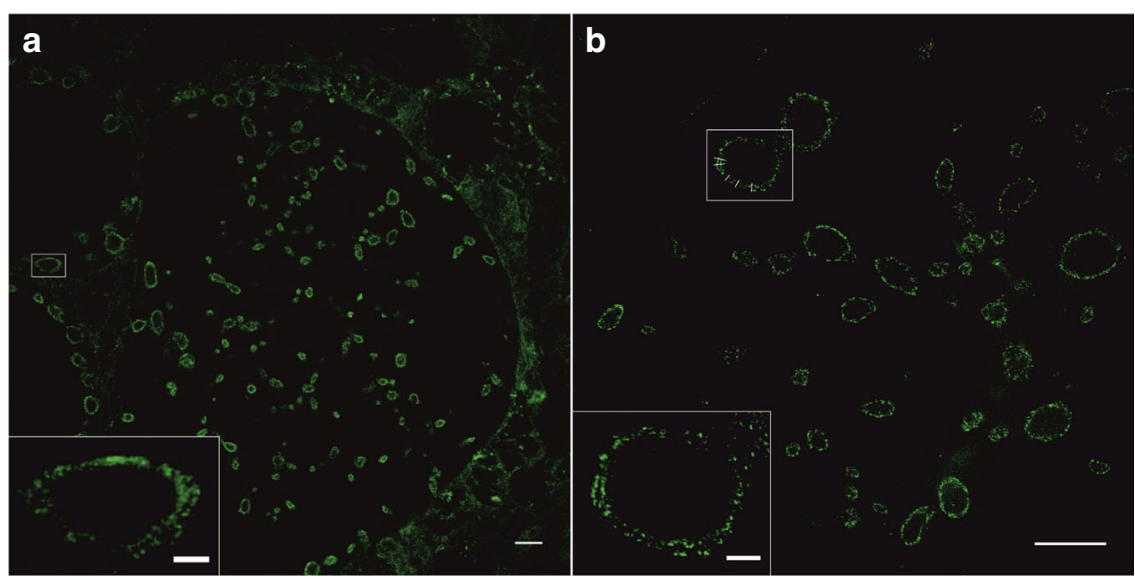

Fig. 4 10x Sphingolipid ExM in combination with SIM resolves the distance between the OM and IM of gram-negative bacteria. HeLa229 cells infected with Chlamydia trachomatis for $24 \mathrm{~h}$, fed with $\alpha-\mathrm{NH}_{2}-\omega-\mathrm{N}_{3}-\mathrm{C}_{6}$-ceramide, fixed, permeabilized and click-labeled with DBCO-Alexa Fluor 488 (green). Confocal (a) and SIM images (b) disclose that ceramides are incorporated into the OM and IM. Fitting intensity cross sectional profiles at different positions by a bimodal Gaussian fit resulted in a peak-to-peak distance of $27.6 \pm 7.7 \mathrm{~nm}$ (s.d.) (Supplementary Figs. 22 and 23). The data were obtained from $n=3$ independent experiments. Scale bars $10 \mu \mathrm{m}(\mathbf{a}, \mathbf{b}), 2 \mu \mathrm{m}$ (white boxes)

polymer ${ }^{9}$. Sphingolipid ExM allows for simultaneous superresolution imaging of membranes and associated proteins in $4 \mathrm{x}$ and 10x expanded samples. In combination with SIM, sphingolipid ExM enables 10-20 nm spatial resolution, approaching that of electron microscopy and has allowed us to resolve details of sphingolipid-protein interactions. Such high spatial resolutions are difficult to achieve using pre-expansion immunolabeling with primary and secondary antibodies but feasible using small membrane incorporated ceramides that are linked into the polymer and fluorescently labeled with minimal linkage error. For clarification, pre-expansion immunolabeling introduces a linkage error of $\sim 17.5 \mathrm{~nm}^{7}$, which translates into a linkage error of $\sim 175$ $\mathrm{nm}$ after 10x expansion. Such large linkage errors in expanded samples severely blur the underlying structure and impede superresolution imaging with high spatial resolution. We hypothesize that our approach of introducing a primary amino group for fixation and linkage into acrylamide polymers by GA can be broadly used to enable ExM of other lipids and thus far inaccessible molecule classes including carbohydrates.

\section{Methods}

A step-by-step protocol describing the expansion of cellular and bacterial membranes can be found at Protocol Exchange ${ }^{48}$.

\section{Chemical synthesis of $\alpha$-amino- $\omega$-azido- $\mathrm{C}_{6}$-ceramide. Starting from $\mathrm{N}$-Boc-} protected L-lysine (1) the introduction of the azide-functionality was accomplished via catalytic diazotransfer reaction to obtain azido-acid 2 in $85 \%$ yield (Fig. 1a). For that, triflyl azide was prepared based on a method of Yan et al. with a reduced amount of highly toxic sodium azide and triflyl anhydride compared to previous protocols ${ }^{49}$. Subsequent amide coupling of 2 with sphingosine was performed in DMF under basic conditions using HATU as coupling reagent. The resulting Bocprotected azido-ceramide analog 3 was isolated in $48 \%$ yield. In the last step the amine group was deprotected by the treatment with TFA in dichloromethane. After basic workup, followed by column chromatography, the target ceramide analog 4 was successfully isolated in 39\% yield (Fig. 1a). Details on the experimental procedures can be found in the Supporting Information. All isolated compounds were characterized by a combination of HRMS, NMR and IR spectroscopy (Supplementary Figs. 1-13).

Cell lines and bacteria. Human HeLa229 cells (ATCC CCL-2.1tm) and human epithelial conjunctival cells (Chang) were cultured in $10 \%(\mathrm{v} / \mathrm{v})$ heat inactivated FBS (Sigma-Aldrich) RPMI1640 + GlutaMAXtm medium (Gibcotm) and were grown in a humidified atmosphere containing $5 \%(\mathrm{v} / \mathrm{v}) \mathrm{CO}_{2}$ at $37^{\circ} \mathrm{C}$. HeLa229 cells were used for infection with Chlamydia trachomatis and Simkania negevensis, Chang cells for infection with Neisseria gonorrhoeae. For this study, C. trachomatis serovar L2/434/Bu (ATCC VR-902B ${ }^{\mathrm{tm}}$ ), S. negevensis and N. gonorrhoeae (strain MS11, derivative N927) were used. C. trachomatis and S. negevensis were propagated in HeLa229 cells at a multiplicity of infection (MOI) of 1 for $48 \mathrm{~h}$ for
C. trachomatis and $72 \mathrm{~h}$ for S. negevensis. The cells were then detached and lysed using glass beads ( $3 \mathrm{~mm}$, Roth). Low centrifugation supernatant (10 $\mathrm{min}$ at $2000 \mathrm{~g}$ at $4{ }^{\circ} \mathrm{C}$ for C. trachomatis and 10 minutes at $600 \mathrm{~g}$ at $4^{\circ} \mathrm{C}$ for S. negevensis) was transferred to high speed centrifugation $\left(30 \mathrm{~min}\right.$ at $30.000 \mathrm{~g}$ at $4^{\circ} \mathrm{C}$ for C. trachomatis and $30 \mathrm{~min}$ at $20.000 \mathrm{~g}$ at $4{ }^{\circ} \mathrm{C}$ for $\mathrm{S}$. negevensis) to pellet the bacteria. Afterwards, the pellet was washed and resuspended in 1x SPG buffer (7.5\% sucrose, $0.052 \% \mathrm{KH}_{2} \mathrm{PO}_{4}, 0.122 \% \mathrm{NaHPO}_{4}, 0.072 \% \mathrm{~L}$-glutamate). The resuspended bacteria were then stored at $-80^{\circ} \mathrm{C}$ and titrated for an MOI of 1 for further experimentation. Infected cells were incubated in a humidified atmosphere with $5 \%(\mathrm{v} / \mathrm{v})$ $\mathrm{CO}_{2}$ at $35^{\circ} \mathrm{C}$. For secondary infections, Hela229 cells were infected with C. trachomatis for 48 hours and then lysed using glass beads ( $3 \mathrm{~mm}$, Roth). Afterwards, the supernatant was diluted 1:100 to infect other cells. The cell lines as well as the Chlamydia used in this study were tested to be free of Mycoplasma via PCR. Neisseria were cultivated on gonococci (GC) agar (ThermoScientific, Waltham, USA) plates supplemented with $1 \%$ vitamin mix at $37^{\circ} \mathrm{C}$ and $5 \% \mathrm{CO}_{2}$ for $16 \mathrm{~h}$. On the day of infection, liquid culture was performed in protease-peptone medium (PPM) supplemented with $1 \%$ vitamin mix and $0.5 \%$ sodium bicarbonate $8.4 \%$ solution $(\mathrm{PPM}+)$ at $37^{\circ} \mathrm{C}$ and $120 \mathrm{rpm}$. Gonococci were grown to an $\mathrm{OD}_{550} 0.4$ to 0.6 . Before infecting the cells, the medium of the liquid culture was changed to 4-(2-Hydroxyethyl)piperazine-1-ethanesulfonic acid (HEPES buffer) medium by centrifugation with $2778 \mathrm{~g}$ for $5 \mathrm{~min}$. After the indicated time of $4 \mathrm{~h}$, the infection was stopped by washing the cells three times with Hepes medium.

Western blot. Western Blot lysates were collected on ice by lysing the cells in SDS sample buffer $(62.5 \mathrm{mM}$ Tris, pH 6.8, 2\% SDS, $20 \%$ glycerol and $5 \% \beta$-mercaptoethanol) and then cooked for $5 \mathrm{~min}$ at $95^{\circ} \mathrm{C}$. The protein samples were separated in $10 \%$ SDS-PAGE gel and then transferred to a PVDF membrane (Roche) in a semi-dry electroblotter. After transfer, the membrane was blocked for $1 \mathrm{~h}$ in Trisbuffer containing $0.05 \%$ Tween 20 and 5\% dry milk powder and afterwards incubated in primary antibody over night at $4{ }^{\circ} \mathrm{C}$. The primary antibodies used were: cHSP60 (Santa Cruz, sc-57840, dilution 1:1000) and $\beta$-actin (Sigma, A5441, dilution 1:10,000). Proteins were detected with secondary antibodies coupled to horseradish peroxidase (Santa Cruz Bioscience) using the ECL system (Pierce) on an Intas Chem HR 16-3200 reader.

LDH assay. LDH-assays were performed using the Cytotoxicity Detection KitPLUS (LDH) (Sigma). For this, Hela229 cells were treated with $10 \mu \mathrm{M} \mathrm{C}_{6}$-Cer, $\omega$ $\mathrm{N}_{3}-\mathrm{C}_{6}$-Cer, $\alpha-\mathrm{NH}_{2}-\omega-\mathrm{N}_{3}-\mathrm{C}_{6}$-ceramide and the controls with $10 \mu \mathrm{l}$ DMSO for 1 or $24 \mathrm{~h}$ in 12 -well plates. Additionally, one control sample was treated with $20 \mu \mathrm{L}$ Lysis Solution for $10 \mathrm{~min}$ at $37^{\circ} \mathrm{C}$. Afterwards, $500 \mu \mathrm{l}$ of the cells supernatant was centrifuged at $14.000 \mathrm{~g} .100 \mu \mathrm{l}$ of the centrifuged supernatant was then transferred to a 96-well plate and incubated with $100 \mu \mathrm{l}$ of a 1:45 mixture of Catalyst (Diaphorase/ $\mathrm{NAD}+$ mixture) and Dye-solution (INT and sodium lactate). The reaction was performed for $15 \mathrm{~min}$ in the dark and then stopped with $50 \mu \mathrm{l}$ of the Stop Solution. The light absorbance of the samples was then measured on a TECAN infinite M200 and compared to the DMSO treated (low control) and the DMSO and Lysis Solution treated (max control) control samples.

Chemistry and immunolabeling. For immunostaining, cells were seeded on $15 \mathrm{~mm}$ coverslips. $a$-amino- $\omega$-azido- $\mathrm{C}_{6}$-ceramide, $\omega$-azido- $\mathrm{C}_{6}$-ceramide, as well as $\omega$-azido-sphingosine were fed with $10 \mu \mathrm{M}$ final concentration for $1 \mathrm{~h}$ at $37^{\circ} \mathrm{C}$. For chlamydial infection, the cells were fed with ceramide-analogs $23 \mathrm{~h}$ post infection 
and for infection with Simkania for $72 \mathrm{~h}$ and for neisserial infection, the cells were fed with the sphingosine analog immediately before infection. Afterwards, the cells were fixed in $4 \%$ PFA and $0.1 \%$ GA for 15 min, washed $3 x$ in $1 x P B S$ and then permeabilized for $15 \mathrm{~min}$ in $0.2 \%$ Triton X-100 in PBS. The cells were then washed again $3 \mathrm{x}$ in $1 \mathrm{xPBS}$ and then incubated with $5 \mu \mathrm{M}$ DBCO-488 (Jena Bioscience, CLK-1278-1) at $37^{\circ} \mathrm{C}$ for $30 \mathrm{~min}$ or $5 \mu \mathrm{M}$ Click-IT Alexa Fluor ${ }^{\circledast} 488$ DIBO alkyne dye (ThermoScientific, Waltham, USA) at $37^{\circ} \mathrm{C}$ for $30 \mathrm{~min}$. For staining with antibodies, the cells were washed, blocked using $2 \%$ FCS in $1 \times$ PBS for $1 \mathrm{~h}$ and then incubated in primary antibody diluted in blocking buffer for $1 \mathrm{~h}$ in a humid chamber. The primary antibodies used in this study were: anti-HSP60 ms (Santa Cruz, sc-57840, dilution 1:200), anti-Neisseria gonorrhoeae primary antibody $\mathrm{rb}$ (US biological, dilution 1:200), anti-Prx3 (Origene, TA322470, dilution 1:100), anti-CERT (Abcam, ab72536, 1:100) and anti-LPS (BioRAD, MCA2718, dilution 1:200). After that, the cells were washed $3 \mathrm{x}$ in $1 \times$ PBS and then incubated in the corresponding secondary antibody diluted in blocking buffer for $1 \mathrm{~h}$ and then washed $3 \mathrm{x}$ with $1 \mathrm{x}$ PBS. The secondary antibodies used were: ATTO $647 \mathrm{~N} \mathrm{~ms}$ (Rockland, 610-156-121 S, dilution 1:200) and ATTO $647 \mathrm{~N} \mathrm{rb}$ (Sigma, 40839, dilution 1:200).

mCling. $150 \mathrm{nmol}$ mCling was incubated in 3 molar excess of ATTO 643 Maleimide (ATTO-TEC, AD 643-45) in $100 \mathrm{mM}$ TCEP overnight at RT under continuous shaking. The label product was purified by HPLC (JASCO) and the concentration was determined using a UV-vis spectrophotometer (Jasco V-650). Staining with mCling was performed by the incubation of living cells in $0.5 \mu \mathrm{M}$ mCling dissolved in media for $10 \mathrm{~min}$ at $37^{\circ} \mathrm{C}$.

Expansion microscopy. Stained cells were treated for 10 min with $0.25 \%$ GA at RT and gelated after three washing steps. In case of $4 \mathrm{x}$ expansion a monomer solution consisting of $8.625 \%$ sodium acrylate (Sigma, 408220 ), 2.5\% acrylamide (Sigma, A9926), 0.15\% N,N'-methylenbisacrylamide (Sigma, A9926), $2 \mathrm{M} \mathrm{NaCl}$ (Sigma, S5886) and $1 \times$ PBS and $0.2 \%$ freshly added ammonium persulfate (APS, Sigma, A3678) and tetramethylethylenediamine (TEMED, Sigma, T7024) was used. Here gelation was performed for $1 \mathrm{~h}$ at RT followed by proteinase digestion. In case of 10x expansion $1 \mathrm{ml}$ of the monomer solution containing $0.267 \mathrm{~g}$ DMAA (Sigma, 274135 ) and $0.064 \mathrm{~g}$ sodium acrylate (Sigma, 408220) dissolved in $0.57 \mathrm{~g} \mathrm{dd \textrm {H } _ { 2 } \mathrm { O }}$ was degassed for $45 \mathrm{~min}$ on ice with nitrogen followed by the addition of $100 \mu \mathrm{l}$ KPS $(0.036 \mathrm{~g} / \mathrm{ml}$, Sigma, 379824). After another $15 \mathrm{~min}$ of degassing and the addition of $4 \mu \mathrm{l}$ TEMED per $\mathrm{ml}$ monomer solution, gelation was performed for 30 $\mathrm{min}$ at RT followed by an incubation of $1.5 \mathrm{~h}$ at $37^{\circ} \mathrm{C}$. Hereafter the samples were digested for $3 \mathrm{~h}$ - overnight in digestion buffer $(50 \mathrm{mM}$ Tris $\mathrm{pH}$ 8.0, $1 \mathrm{mM}$ EDTA (Sigma, ED2P), 0.5\% Triton X-100 (Thermo Fisher, 28314) and $0.8 \mathrm{M}$ guanidine $\mathrm{HCl}$ (Sigma, 50933)), supplied with $8 \mathrm{U} / \mathrm{ml}$ protease K (Thermo Fisher, AM2548) and for expansion of Neisseria additional $1 \mathrm{mg} / \mathrm{ml}$ Lysozyme according to Lim et al. ${ }^{50}$. Digested gels were expanded in hourly changed $\mathrm{ddH}_{2} \mathrm{O}$ until the expansion saturated. The expansion factor was determined by the gel size using calipers directly after gelation and by the gel size of the digested and expanded samples. We achieved experimental expansion factors of 4.1 for the $4 \mathrm{x}$ monomer solution and 10 for the 10x monomer solution, and the expansion factor remained constant for the used monomer solutions. Expanded and chopped gels were stored at $4{ }^{\circ} \mathrm{C}$ in $\mathrm{ddH}_{2} \mathrm{O}$ immobilized prior to imaging on PDL-coated glass chambers (Merck, 7342055).

Confocal microscopy and SIM. Confocal imaging was performed on an inverted microscope (Zeiss LSM700 using software ZEN 12.0.1.362, 2012) or on a Leica TCS SP5 confocal microscope (Leica Biosystems using software LAS AF version 2.7.3.9723) and SIM-imaging on a Zeiss ELYRA S.1 SR-SIM structured illumination platform using a $63 \mathrm{x}$ water-immersion objective (C-Apochromat, $63 \times 1.2 \mathrm{NA}$, Zeiss, 441777-9970). Reconstruction of SIM-images was performed using the ZEN image-processing platform with a SIM module. Z-stacks were processed using Imaris 8.4.1 and FIJI $1.51 \mathrm{n}^{51}$.

FRAP. HeLa229 cells were seeded in an 8-well chambered high precision coverglass (Sarstedt 8-well on coverglass II) and incubated for $24 \mathrm{~h}$ at $37^{\circ} \mathrm{C}$ and $5 \% \mathrm{CO}_{2}$. The cells were fed with $10 \mu \mathrm{M}$ of the corresponding azido-ceramide analog for $30 \mathrm{~min}$ in cell culture media. Afterwards, the cells were washed with HBSS with magnesium and calcium and fixed with $4 \%$ formaldehyde and $0.1 \%$ glutaraldehyde in HBSS for $15 \mathrm{~min}$ at room temperature and washed. Ceramides were labeled by strain-promoted alkyne-azide cycloaddition (SPAAC) with $10 \mu \mathrm{M}$ DBCO-Alexa Fluor 488 in HBSS for $30 \mathrm{~min}$ at $37^{\circ} \mathrm{C}$ and washed. FRAP-imaging was performed at a confocal laser scanning microscope (CLSM) LSM700 (Zeiss, Germany) using the Plan-Apochromat $63 \times 1.4$ oil objective. Using the $488 \mathrm{~nm}$ laser line as excitation, a time series with 30 frames every $1.5 \mathrm{~s}$ was recorded. After three frames, a circular region of interest with diameter $1.8 \mu \mathrm{m}$ was bleached and fluorescence recovery followed over time.
Reporting summary. Further information on research design is available in the Nature Research Reporting Summary linked to this article.

\section{Data availability}

The data that support the findings of this study are available from the corresponding author upon reasonable request. The source data underlying Fig. $1 \mathrm{~b}$ and Supplementary Figs. 18c and 19, and 26 are provided as a Source Data file. The raw main Figure files are deposited at https://doi.org/10.6084/m9.figshare.13147955. Source data are provided with this paper.

Received: 8 June 2020; Accepted: 5 November 2020;

Published online: 02 December 2020

\section{References}

1. Sauer, M. \& Heilemann, M. Single-molecule localization microscopy in eukaryotes. Chem. Rev. 117, 7478-7509 (2017).

2. Schermelleh, L. et al. Super-resolution microscopy demystified. Nat. Cell Biol. 21, 72-84 (2019).

3. Chen, F., Tillberg, P. W. \& Boyden, E. S. Optical imaging. Expansion Microsc Sci. 347, 543-548 (2015).

4. Wassie, A. T., Zhao, Y. \& Boyden, E. S. Expansion microscopy: principles and uses in biological research. Nat. Methods 16, 33-41 (2019).

5. Truckenbrodt, S., Sommer, C., Rizzoli, S. O. \& Danzl, J. G. A practical guide to optimization in X10 expansion microscopy. Nat. Protoc. 14, 832-863 (2019).

6. Zwettler, F. U. et al. Molecular resolution imaging by post-labeling expansion single-molecule localization microscopy (Ex-SMLM). Nat. Commun. 11, 3388 (2020)

7. Chang, J. B. et al. Iterative expansion microscopy. Nat. Methods 14, 593-599 (2017).

8. Kunz, T. C., Gotz, R., Sauer, M. \& Rudel, T. Detection of chlamydia developmental forms and secreted effectors by expansion microscopy. Front Cell Infect. Microbiol 9, 276 (2019).

9. Chozinski, T. J. et al. Expansion microscopy with conventional antibodies and fluorescent proteins. Nat. Methods 13, 485-488 (2016).

10. Tillberg, P. W. et al. Protein-retention expansion microscopy of cells and tissues labeled using standard fluorescent proteins and antibodies. Nat. Biotechnol. 34, 987-992 (2016).

11. Chen, F. et al. Nanoscale imaging of RNA with expansion microscopy. Nat. Methods 13, 679-684 (2016).

12. Gambarotto, D. et al. Imaging cellular ultrastructures using expansion microscopy (U-ExM). Nat. Methods 16, 71-74 (2019).

13. Zwettler, F. U. et al. Tracking down the molecular architecture of the synaptonemal complex by expansion microscopy. Nat. Commun. 11, 3222 (2020).

14. Sheard, T. M. D. et al. Three-dimensional and chemical mapping of intracellular signaling nanodomains in health and disease with enhanced expansion microscopy. ACS Nano 13, 2143-2157 (2019).

15. Koch-Edelmann, S. et al. The cellular ceramide transport protein CERT promotes Chlamydia psittaci infection and controls bacterial sphingolipid uptake. Cell Microbiol. 19, e12752 (2017).

16. Derre, I., Swiss, R. \& Agaisse, H. The lipid transfer protein CERT interacts with the Chlamydia inclusion protein IncD and participates to ER-Chlamydia inclusion membrane contact sites. PLoS Pathog. 7, e1002092 (2011).

17. Fink, J. \& Seibel, J. Click reactions with functional sphingolipids. Biol. Chem. 399, 1157-1168 (2018).

18. Bécam, J. et al. Antibacterial activity of ceramide and ceramide analogs against pathogenic Neisseria. Sci. Rep. 7, 17627 (2017).

19. Hannun, Y. A. \& Obeid, L. M. The Ceramide-centric universe of lipidmediated cell regulation: stress encounters of the lipid kind. J. Biol. Chem. 277, 25847-25850 (2002).

20. Burgert, A. et al. Characterization of plasma membrane ceramides by superresolution microscopy. Angew. Chem. Int. Ed. Engl. 56, 6131-6135 (2017)

21. Walter, T. et al. Incorporation studies of clickable ceramides in Jurkat cell plasma membranes. Chem. Commun. 53, 6836-6839 (2017).

22. Walter, T. et al. Incorporation and visualization of azido-functionalized Noleoyl serinol in Jurkat cells, mouse brain astrocytes, 3T3 fibroblasts and human brain microvascular endothelial cells. Chem. Commun. 52, 8612-8614 (2016).

23. Collenburg, L. et al. Functionalized sphingolipid analogue for studying redistribution during activation in living T cells. J. Immunol. 196, 3951-3962 (2016).

24. Revelo, N. H. et al. A new probe for super-resolution imaging of membranes elucidates trafficking pathways. J. Cell Biol. 205, 591-606 (2014) 
25. Karagiannis, E. D., et al. IMAXT Grand Chellenge Consortium, Marblestone, A. H., Kasthuri, N., Boyden, E. S. Expansion Microscopy of Lipid Membranes. Preprint at https://doi.org/10.1101/829903 (2019).

26. Wen, G. et al. Evaluation of direct grafting strategies via trivlanet anchoring for enabling lipid membrane and cytoskeleton staining in expansion microscopy. ACS Nano 14, 7860-7867 (2020).

27. Grassme, H., Riethmuller, J. \& Gulbins, E. Biological aspects of ceramideenriched membrane domains. Prog. Lipid Res. 46, 161-170 (2007).

28. Gulbins, E., Dreschers, S., Wilker, B. \& Grassme, H. Ceramide, membrane rafts and infections. J. Mol. Med. 82, 357-363 (2004).

29. Faulstich, M. et al. Neutral sphingomyelinase 2 is a key factor for PorB-dependent invasion of Neisseria gonorrhoeae. Cell Microbiol. 17, 241-253 (2015).

30. Herweg, J. A. et al. Proteomic analysis of the Simkania-containing vacuole: the central role of retrograde transport. Mol. Microbiol. 99, 151-171 (2016).

31. Hackstadt, T., Rockey, D. D., Heinzen, R. A. \& Scidmore, A. Chlamydia trachomatis interrupts an exocytic pathway to acquire endogenously synthesized sphingomyelin in transit from the Golgi apparatus to the plasma membrane. EMBO J. 15, 964-977 (1996)

32. Kunz, T. C. \& Kozjak-Pavlovic, V. Diverse facets of sphingolipid involvement in bacterial infections. Front. Cell Dev. Biol. 7, 203 (2019).

33. Belland, R., Ojcius, D. M. \& Byrne, G. I. Chlamydia. Nat. Rev. Microbiol. 2, 530-531 (2004).

34. Banhart, S., Schafer, E. K., Gensch, J. M. \& Heuer, D. Sphingolipid metabolism and transport in Chlamydia trachomatis and Chlamydia psittaci infections. Front. Cell Dev. Biol. 7, 223 (2019).

35. Hanada, K. Intracellular trafficking of ceramide by ceramide transfer protein. Proc. Jpn. Acad. Ser. B Phys. Biol. Sci. 86, 426-437 (2010).

36. Zeidan, Y. H. \& Hannun, Y. A. Translational aspects of sphingolipid metabolism. Trends Mol. Med. 13, 327-336 (2007).

37. Nguyen, B. D. et al. Lipooligosaccharide is required for the generation of infectious elementary bodies in Chlamydia trachomatis. Proc. Natl Acad. Sci. USA 108, 10284-10289 (2011).

38. Fischer, C. L. et al. Antibacterial activity of sphingoid bases and fatty acids against Gram-positive and Gram-negative bacteria. Antimicrob. Agents Chemother. 56, 1157-1161 (2012).

39. Solger, F. et al. A role of shingosine in the intracellular survival of Neisseria gonorrhoeae. Front. Cell. Infect. Microbiol. 10, 215 (2020).

40. Lee, J. K. et al. Replication-dependent size reduction precedes differentiation in Chlamydia trachomatis. Nat. Commun. 9, 45 (2018)

41. Gustafsson, M. G. Surpassing the lateral resolution limit by a factor of two using structured illumination microscopy. J. Microsc. 198, 82-87 (2000).

42. Hoare, A., Timms, P., Bavoil, P. M. \& Wilson, D. P. Spatial constraints within the chlamydial host cell inclusion predict interrupted development and persistence. BMC Microbiol. 8, 5 (2008).

43. Auer, D., Hugelschaffer, S. D., Fischer, A. B. \& Rudel, T. The chlamydial deubiquitinase Cdul supports recruitment of Golgi vesicles to the inclusion. Cell. Microbiol. 22, e13136 (2019).

44. Elwell, C. A. et al. Chlamydia trachomatis co-opts GBF1 and CERT to acquire host sphingomyelin for distinct roles during intracellular development. PLoS Pathog. 7, e1002198 (2011).

45. Asmar, A. T. et al. Communication across the bacterial cell envelope depends on the size of the periplasm. PLoS Biol. 15, e2004303 (2017).

46. Ghai, S. Understanding antibiotic resistance via outer membrane permeability. Infect. Drug Resist. 11, 523-530 (2018).

47. $\mathrm{Wu}, \mathrm{D}$. et al. Aging up-regulates expression of inflammatory mediators in mouse adipose tissue. J. Immunol. 179, 4829-4839 (2007).

48. Götz, R. et al. Expansion microscopy of cellular and bacterial membranes byfunctionalized ceramides. Protoc. Exchange. https://doi.org/10.21203/rs.3. pex-1249/v1 (2020).
49. Yan, R.-B., Yang, F., Wu, Y., Zhang, L.-H. \& Ye, X.-S. An efficient and improved procedure for preparation of triflyl azide and application in catalytic diazotransfer reaction. Tetrahedron Lett. 46, 8993-8995 (2005).

50. Lim, Y. et al. Mechanically resolved imaging of bacteria using expansion microscopy. PLoS Biol. 17, e3000268 (2019).

51. Schindelin, J. et al. Fiji: an open-source platform for biological-image analysis. Nat. Methods 9, 676-682 (2012).

\section{Acknowledgements}

We thank Elke Maier for the preparation of S. negevensis stocks. This work was supported by the Deutsche Forschungsgemeinschaft (DFG) GRK 2157 to V.K.P., T.R. and M.S., and DFG FOR 2123 to T.R. and M.S.

\section{Author contributions}

The manuscript was written through contributions of all authors. R.G. and T.C.K. designed and performed experiments, analyzed data and wrote the manuscript. J.F. and J.S. synthesized $\alpha$-amino- $\omega$-azido-C6-ceramide, J.Sch., F.S. and V.K-P. performed experiments. T.R. and M.S. designed the experiments and wrote the manuscript. All authors have given approval to the final version of the manuscript.

\section{Funding}

Open Access funding enabled and organized by Projekt DEAL.

\section{Competing interests}

The authors declare no competing interests.

\section{Additional information}

Supplementary information is available for this paper at https://doi.org/10.1038/s41467 020-19897-1.

Correspondence and requests for materials should be addressed to T.R. or M.S.

Peer review information Nature Communications thanks the anonymous reviewer(s) for their contribution to the peer review of this work. Peer reviewer reports are available.

Reprints and permission information is available at http://www.nature.com/reprints

Publisher's note Springer Nature remains neutral with regard to jurisdictional claims in published maps and institutional affiliations.

Open Access This article is licensed under a Creative Commons Attribution 4.0 International License, which permits use, sharing, adaptation, distribution and reproduction in any medium or format, as long as you give appropriate credit to the original author(s) and the source, provide a link to the Creative Commons license, and indicate if changes were made. The images or other third party material in this article are included in the article's Creative Commons license, unless indicated otherwise in a credit line to the material. If material is not included in the article's Creative Commons license and your intended use is not permitted by statutory regulation or exceeds the permitted use, you will need to obtain permission directly from the copyright holder. To view a copy of this license, visit http://creativecommons.org/ licenses/by/4.0/

(C) The Author(s) 2020 